Supporting Information

For

\title{
Catalytic Allylic Oxidation with a Recyclable, Fluorous
}

\section{Seleninic Acid}

\section{David Crich* and Yekui Zou}

Department of Chemistry, University of Illinois at Chicago, 845 West Taylor Street, Chicago, IL 60607-7061. 


\section{Table of Contents}

\begin{tabular}{|l|c|}
\hline Compound & Page \\
\hline Dibutyl diselenide & $\mathrm{S}-3$ \\
\hline Butyl perfluorooctyl selenide & $\mathrm{S}-3$ \\
\hline Perfluorooctyl seleninic acid & $\mathrm{S}-4$ \\
\hline 3 $\beta$-Benzoyloxycholest-5-ene-4-one (10) & $\mathrm{S}-5$ \\
\hline 2-Phenyl-2-cyclohexen-1-one (12) & $\mathrm{S}-5$ \\
\hline 5-Decen-4-one (14) & $\mathrm{S}-5$ \\
\hline 8-Acetoxy-2,6-dimethyldeca-2E, 6E-dien-1-al (16) & $\mathrm{S}-5$ \\
\hline 2-Cycloocten-1-one (18) & $\mathrm{S}-6$ \\
\hline 6,6-Dimethylenebicyclo[3,1,1]heptan-3-one (20) & $\mathrm{S}-6$ \\
\hline 6,6-Dimethyl-bicyclo[3.1.1]hept-2-ene-2-carbaldehyde (22) & $\mathrm{S}-6$ \\
\hline
\end{tabular}




\section{Preparation of catalyst}

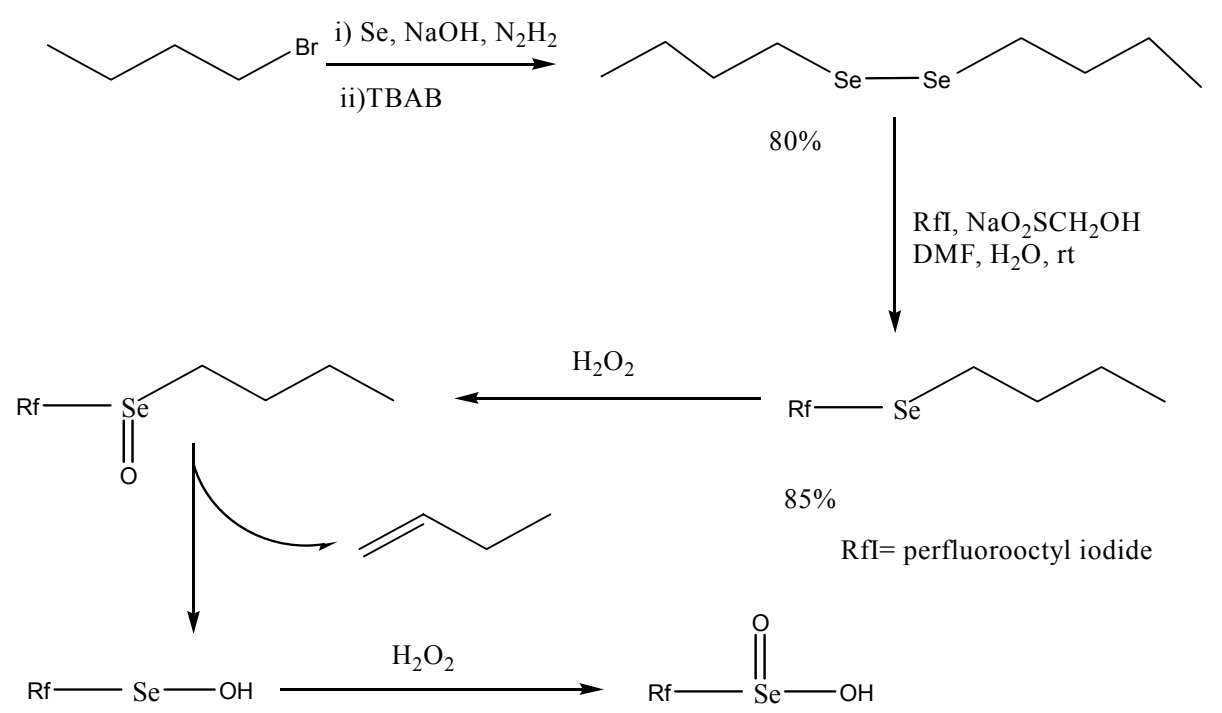

Preparation of dibutyl diselenide. ${ }^{1}$ To a mixture of selenium powder $(2.0 \mathrm{~g}, 25 \mathrm{mmol})$ and sodium hydroxide $(1.0 \mathrm{~g}, 25 \mathrm{mmol})$ was added $85 \%$ hydrazine hydrate $(0.5 \mathrm{~mL}, 8.5 \mathrm{mmol})$ under nitrogen. The mixture was heated to $70{ }^{\circ} \mathrm{C}$ with stirring for until all the selenium powder was consumed $(3 \mathrm{~h})$. Then TBAB (tetrabutyl ammonium bromide) (0.15 g, $88 \mathrm{mmol}$ ) and butyl bromide (3.4 g, $25 \mathrm{mmol})$ were then added leading to the separation of a heavy oil. The reaction mixture was allowed to cool down to room temperature, the organic layer was separated and the aqueous layer was extracted with petroleum ether $(2 \times 5 \mathrm{~mL})$. The combined organic layer was washed with water $(2 \times 10 \mathrm{~mL})$, then brine $(2 \times 10 \mathrm{~mL})$ and dried over anhydrous sodium sulfate. After concentration of the crude mixture, the residue was distilled $\left(80{ }^{\circ} \mathrm{C}, 1 \mathrm{~mm} \mathrm{Hg}\right)$ to afford dibutyl diselenide ${ }^{2}(2.72 \mathrm{~g}, 80 \%)$ as an oil. ${ }^{1} \mathrm{H}$ NMR $\left(\mathrm{CDCl}_{3}\right) \delta 0.92(\mathrm{t}, J=7.5 \mathrm{~Hz}, 6 \mathrm{H}), 1.39$ (sextet, $J=7.5 \mathrm{~Hz}, 4 \mathrm{H}), 1.71$ (quintet, $J=7.5 \mathrm{~Hz}, 4 \mathrm{H}), 2.95(\mathrm{t}, J=7.5 \mathrm{~Hz} 4 \mathrm{H}) ;{ }^{13} \mathrm{C}: \delta 13.6,22.6$, $30.0,33.1$.

Preparation of butyl perfluorooctyl selenide (6). ${ }^{3}$ Dibutyl diselenide $(1.20 \mathrm{~g}, 4.41 \mathrm{mmol})$ was dissolved in DMF (15.0 mL) under nitrogen. Perfluorooctyl iodide (2.36 mL, $8.80 \mathrm{mmol})$, 
prewashed with a $10 \%$ aqueous solution of $\mathrm{Na}_{2} \mathrm{~S}_{2} \mathrm{O}_{5}$, was added followed by water $(0.5 \mathrm{~mL})$. Lastly sodium hydroxymethanesulfinate $(2.04 \mathrm{~g}, 13.2 \mathrm{mmol})$ was added in portions over $3 \mathrm{~h}$. The solution was stirred at room temperature for $20 \mathrm{~h}$, then the mixture was extracted with $\mathrm{Et}_{2} \mathrm{O}$ and the organic layer washed with $5 \%$ aqueous $\mathrm{NaHCO}_{3}$ and water, then dried over $\mathrm{Na}_{2} \mathrm{SO}_{4}$ and concentrated under reduced pressure. The product was purified by flash chromatography on silica gel using hexane as eluent to afford butyl perfluorooctyl selenide $(4.15 \mathrm{~g}, 85 \%$ based on $\left.\mathrm{C}_{8} \mathrm{~F}_{17} \mathrm{I}\right)$ in the form of an oil. ${ }^{1} \mathrm{H}$ NMR $\left(\mathrm{CDCl}_{3}\right) \delta 0.94(\mathrm{t}, J=7.5 \mathrm{~Hz}, 3 \mathrm{H}), 1.43$ (sextet, $J=7.5$ $\mathrm{Hz}, 2 \mathrm{H}$ ), 1.76 (quintet, $J=7.5 \mathrm{~Hz}, 2 \mathrm{H}$ ), $3.03(\mathrm{t}, J=7.5 \mathrm{~Hz}, 2 \mathrm{H}) ;{ }^{19} \mathrm{~F}$ NMR $\delta 8.4,-14.4,-45.9$, $48.9,-49.5,-50.4,-53.8$; HRMS $\left[\mathrm{M}^{\bullet+}\right] 555.9598$, found 555.9559 .

Preparation of perfluorooctylseleninic acid (5). Hydrogen peroxide (30\%, $10.00 \mathrm{mmol}, 1.00$ $\mathrm{mL})$ was added to a stirred solution of butyl perfluorooctyl selenide $(0.56 \mathrm{~g}, 1.00 \mathrm{mmol})$ in hexafluoroisopropanol $(8.00 \mathrm{~mL})$ in a closed flask at $0{ }^{\circ} \mathrm{C}$, and then the reaction mixture was warmed to room temperature and stirred for $20 \mathrm{~h}$. Perfluorooctylseleninic acid $(0.49 \mathrm{~g}, 92 \%)$ was obtained as an amorphous white solid after evaporation of the solvent. M.p. $135-137{ }^{\circ} \mathrm{C}$; HRMS [M-H] $]^{-}$530.8799, found 530.8792. The insoluble nature of this acid in most solvents precluded the measurement of NMR spectroscopic data.

General procedure for Allylic oxidation. The olefin $(1.0 \mathrm{mmol})$, iodoxybenzene $(3.0 \mathrm{mmol})$ and perfluorooctyl seleninic acid $(0.1 \mathrm{mmol})$ were stirred in anhydrous $\alpha, \alpha, \alpha$-trifluorotoluene $(10.0 \mathrm{~mL})$ at reflux under nitrogen. After complete conversion of the substrate, the mixture was allowed to cool to room temperature. Sodium metabisulfite $(1.0 \mathrm{mmol})$ was then added and the mixture stirred for $3 \mathrm{~h}$. The reaction mixture was then diluted with ethyl acetate $(8.0 \mathrm{~mL})$, and the organic phase was washed with water and brine and concentrated in vacuo to give a residue 
which was portioned between $\mathrm{CH}_{2} \mathrm{Cl}_{2}(3.0 \mathrm{~mL})$ and $\mathrm{FC}-72(20.0 \mathrm{~mL})$ in the water-cooled continuous extractor ${ }^{4}$ for $3 \mathrm{~h}$. Evaporation of the FC-72 phase provided the recovered diselenide whereas the organic phase afforded the enone after filtration on silica gel.

3及-Benzoyloxycholest-5-ene-4-one (10). ${ }^{5}$ Oxidation of $3 \beta$-cholesteryl benzoate $(0.49 \mathrm{~g}, 1.0$ mmol) and subsequent chromatography on silica gel (eluant: dichloromethane-hexane 3:1) afforded 10 (0.33 g, 65\%). M.p. $160-160.5{ }^{\circ} \mathrm{C}$ (acetone); 1 it ${ }^{5}$ m.p. $158-160{ }^{\circ} \mathrm{C} .{ }^{1} \mathrm{H}$ NMR $\left(\mathrm{CDCl}_{3}\right)$ key data: $\delta 5.44(\mathrm{dd}, J=11.9,7.4 \mathrm{~Hz}, 1 \mathrm{H}), 6.41(\mathrm{~m}, 1 \mathrm{H}), 7.44(\mathrm{t}, J=7.2 \mathrm{~Hz}, 2 \mathrm{H}), 7.57(\mathrm{t}, J=7.2$ $\mathrm{Hz}, 1 \mathrm{H}), 8.10$ (d, $J=7.2 \mathrm{~Hz}, 2 \mathrm{H})$. Diperfluorooctyl diselenide (46 mg, 92\%) was recovered from this experiment.

2-Phenyl-2-cyclohexen-1-one (12). ${ }^{6}$ Following the general procedure described for allylic oxidation and chromatography on silica gel (eluant: dichloromethane), 1-phenylcyclohexene (158 mg, $1.0 \mathrm{mmol})$ afforded $12(108 \mathrm{mg}, 63 \%) .{ }^{1} \mathrm{H}$ NMR $\left(\mathrm{CDCl}_{3}\right) \delta 1.97(\mathrm{q}, J=6.3 \mathrm{~Hz}, 2 \mathrm{H})$, $2.4(\mathrm{~m}, 4 \mathrm{H}), 6.88(\mathrm{t}, J=4.4 \mathrm{~Hz}, 1 \mathrm{H}), 7.22$ (br s, 5H). Diperfluorooctyl diselenide (44 mg, 88\%) was recovered from this experiment.

5-Decen-4-one (14). ${ }^{7}$ Oxidation of trans-5-decene (140 $\left.\mathrm{mg}, 1.0 \mathrm{mmol}\right)$ followed by chromatography (eluant: dichloromethane) gave 14 (99 mg, 64\%) as a colorless oil. ${ }^{1} \mathrm{H}$ NMR $\left(\mathrm{CDCl}_{3}\right) \delta 6.1(\mathrm{~d}, J=15.6 \mathrm{~Hz}, 1 \mathrm{H}), 6.8(\mathrm{td}, J=6.8,15.6 \mathrm{~Hz}, 1 \mathrm{H})$. Diperfluorooctyl diselenide (44 mg, 88\%) was recovered from this experiment.

8-Acetoxy-2,6-dimethyldeca-2E,6E-dien-1-al (16). ${ }^{7}$ Oxidation of Geranyl acetate (196 mg, 1.0 $\mathrm{mmol}$ ) and chromatography on silica gel (eluant: dichloromethane) provided 16 (106 $\mathrm{mg}, 50 \%)$. B.p. $160^{\circ} \mathrm{C} / 15 \mathrm{~mm}$ (Kugelrohr); ${ }^{1} \mathrm{H}$ NMR $\left(\mathrm{CDCl}_{3}\right) \delta 1.72$ (s, 6H), 2.04 (s, 3H), 2.22 (t, $J=4.7$ 
$\mathrm{Hz}, 2 \mathrm{H}), 2.50(\mathrm{~m}, 2 \mathrm{H}), 4.58(\mathrm{~d}, J=7.2 \mathrm{~Hz}, 2 \mathrm{H}), 5.37(\mathrm{t}, J=5.7 \mathrm{~Hz}, 1 \mathrm{H}), 6.43(\mathrm{t}, J=6.0 \mathrm{~Hz}, 1 \mathrm{H})$, 9.37 (s, 1H). Diperfluorooctyl diselenide (45 mg, 90\%) was recovered from this experiment.

cis-2-Cycloocten-1-one (18). ${ }^{8}$ Oxidation of cis-cyclooctene (110 mg, $\left.1.0 \mathrm{mmol}\right)$ provided $\mathbf{1 8}$ (72 mg, 58\%). ${ }^{1} \mathrm{H}$ NMR $\left(\mathrm{CDCl}_{3}\right) \delta 1.7 \sim 2.0(\mathrm{~m}, 6 \mathrm{H}), 2.4 \sim 2.6(\mathrm{~m}, 4 \mathrm{H}), 6.28(\mathrm{~d}, J=11.4 \mathrm{~Hz}, 1 \mathrm{H})$, 6.74 (td, $J=9.2,11.4 \mathrm{~Hz}, 1 \mathrm{H})$. Diperfluorooctyl diselenide (45 mg, 90\%) was recovered from this experiment.

6,6-Dimethylenebicyclo[3,1,1] heptan-3-one (20). ${ }^{7}$ Oxidation of $\beta$-pinene $(136 \mathrm{mg}, 1.0 \mathrm{mmol})$ and chromatography on silica gel (eluant: dichloromethane) afforded 20 (62 mg, 41\%). B.p.130 ${ }^{\circ} \mathrm{C} / 15 \mathrm{~mm}$ (Kugelrohr); ${ }^{1} \mathrm{H}$ NMR $\left(\mathrm{CDCl}_{3}\right) \delta 0.81(\mathrm{~s}, 3 \mathrm{H}), 1.36(\mathrm{~s}, 3 \mathrm{H}), 5.01(\mathrm{~d}, J=1.5 \mathrm{~Hz}, 1 \mathrm{H})$, $6.10(\mathrm{~d}, J=1.5 \mathrm{~Hz}, 1 \mathrm{H})$. Diperfluorooctyl diselenide (45 mg, 90\%) was recovered from this experiment.

6,6-Dimethyl-bicyclo[3.1.1] hept-2-ene-2-carbaldehyde (22). ${ }^{9}$ Oxidation of $\alpha$-pinene (136mg, $1.0 \mathrm{mmol}$ ) and chromatography on silica gel (eluant: dichloromethane) provided 22 (93 $\mathrm{mg}$, 62\%). ${ }^{1} \mathrm{H} \mathrm{NMR}\left(\mathrm{CDCl}_{3}\right) \delta 0.71(\mathrm{~s}, 3 \mathrm{H}), 1.31(\mathrm{~s}, 3 \mathrm{H}), \delta 2.87(\mathrm{t}, J=5.9 \mathrm{~Hz}, 1 \mathrm{H}), 6.69(\mathrm{~m}, 1 \mathrm{H})$, $9.41(\mathrm{~s}, 1 \mathrm{H})$. Diperfluorooctyl diselenide (43 mg, 86\%) was recovered from this experiment.

1. Hu, X.B.; Tian, Z.J.; Lu, X.R.; Chen, Y.Y. Synth. Commun, 1997, 27, 553.

2. Salama, P.; Bernard, C. Tetrahedron Lett, 1995, 36, 5571.

3. Magnier, E.; Vit, E.; Wakselman, C. Synlett 2001, 1260.

4. Crich, D.; Hao, X.; Lucas, M. Tetrahedron 1999, 55, 14261.

5. Vigier, A.; Marquet, A.; Barton, D.H.R.; Motherwell, W.B.; Zard, S.Z. J. Chem. Soc., Perkin Trans. 1, 1982, 1937.

6. Reich, H.J.; Renga, J.M.; Reich, I.L. J. Am. Chem. Soc. 1975, 97, 5434.

7. Barton, D.H.R.; Crich, D. Tetrahedron 1985, 41, 4439.

8. House, H.O.; Sieloff, R.F.; Lee, T.V.; DeTar, M.B. J. Org. Chem., 1980, 45, 1800.

9. Kaplan, F.; Schulz, C.O.; Weisleder, D.; Klopfenstein, C. J. Org. Chem., 1968, 33, 1728. 